\title{
Effects of inhomogeneities and thermal fluctuations on the spectral function of a model $d$-wave superconductor
}

\author{
Daniel Valdez-Balderas* and David Stroud ${ }^{\dagger}$ \\ Department of Physics, The Ohio State University, Columbus, Ohio 43210, USA \\ (Received 15 April 2007; revised manuscript received 25 July 2007; published 22 January 2008)
}

\begin{abstract}
We compute the spectral function $A(\mathbf{k}, \omega)$ of a model two-dimensional high-temperature superconductor at both zero and finite temperatures $T$. The model consists of a two-dimensional BCS Hamiltonian with $d$-wave symmetry, which has a spatially varying, thermally fluctuating, complex gap $\Delta$. Thermal fluctuations are governed by a Ginzburg-Landau free energy functional. We assume that an areal fraction $c_{\beta}$ of the superconductor has a large $\Delta$ ( $\beta$ regions), while the rest has a smaller $\Delta$ ( $\alpha$ regions), both of which are randomly distributed in space. We find that $A(\mathbf{k}, \omega)$ is most strongly affected by inhomogeneity near the point $\mathbf{k}$ $=(\pi, 0)$ (and the symmetry-related points). For $c_{\beta} \simeq 0.5, A(\mathbf{k}, \omega)$ exhibits two double peaks (at positive and negative energies) near this $k$ point if the difference between $\Delta_{\alpha}$ and $\Delta_{\beta}$ is sufficiently large in comparison to the hopping integral; otherwise, it has only two broadened single peaks. The strength of the inhomogeneity required to produce a split spectral function peak suggests that inhomogeneity is unlikely to be the cause of a second branch in the dispersion relation, such as has been reported in underdoped LSCO. Thermal fluctuations also affect $A(\mathbf{k}, \omega)$ most strongly near $\mathbf{k}=(\pi, 0)$. Typically, peaks that are sharp at $T=0$ become reduced in height, broadened, and shifted toward lower energies with increasing $T$; the spectral weight near $\mathbf{k}=(\pi, 0)$ becomes substantial at zero energy for $T$ greater than the phase-ordering temperature.
\end{abstract}

DOI: 10.1103/PhysRevB.77.014515

PACS number(s): 74.81.-g, 74.20.De, 74.20.Fg

\section{INTRODUCTION}

In the past several years, the measured electronic properties of cuprate superconductors have shown considerable evidence of inhomogeneities. For example, spatial variations of the superconducting energy gap and of the local density of states spectrum have been observed in scanning tunneling microscopy (STM) experiments. ${ }^{1-5,7,6,8}$ There have also been a number of reports of magnetic and charge orderings in these materials, which also indicate inhomogeneities. ${ }^{9-17}$ Other studies of cuprates have shown that electronic states within certain energy ranges show checkerboardlike spatial modulations. ${ }^{18}$

A number of theoretical approaches have been developed to model these inhomogeneities. ${ }^{19-33}$ These works are reviewed and extended in a recent article. ${ }^{34}$ In the present paper, we use the approach of Ref. 34 to explore how the spectral function of a $d$-wave superconductor is affected by gap inhomogeneities and thermal fluctuations.

The spectral function of cuprate superconductors has been studied theoretically by a number of groups, though most have omitted the effects of quenched inhomogeneities. For example, Wakabayashi et al. ${ }^{35}$ used a weak-coupling BCS theory combined with a Green's function approach to explain the narrow quasiparticle peak at the gap edge, which has been observed by angle-resolved photoemission spectroscopy (ARPES) experiments in overdoped cuprates along the antinodal direction. Pieri et al., ${ }^{36}$ using a Nambu formalism, have studied a model which includes pairing fluctuation effects and which accounts for some features of the singleparticle spectral function as observed in certain cuprates. Zacher et al. ${ }^{37}$ have used a cluster perturbation technique to compute the single-particle spectral function of the $t-J$ and Hubbard models and to study stripe phases in the cuprates. Paramekanti et al. $^{38}$ have studied a Hubbard model for (uni- form) projected $d$-wave states. They used a variational Monte Carlo technique in which one of the variational parameters is the magnitude $\Delta$ of the pairing field and find that $\Delta$, as a function of doping, scales with the $(\pi, 0)$ hump and $T^{*}$ as observed in ARPES experiments.

In another recent work, using a generalization of the BCS theory, Chen et al. ${ }^{39}$ found a sharpening of the peaks in the spectral function as $T$ is reduced below $T_{c}$, similar to what we find as discussed below. (Their model involves a homogeneous superconductor.) Hotta et al. ${ }^{40}$ have used a selfconsistent $t$-matrix approximation to study a model for $s$ - and $d$-wave superconductivities at finite $T$. They found a gap in both the single-particle density of states and the spectral function even above the superconducting transition temperature $T_{c}$; the energy scale for the pseudogap is found to be the Cooper-pair binding energy.

More recently, Mayr et al. ${ }^{41}$ have introduced an extended Hubbard model, which includes both superconductivity and antiferromagnetism; they found that quenched disorder is a necessary ingredient for that model to reproduce the double branch, or split band, observed in ARPES experiments on $\mathrm{La}_{2-x} \mathrm{Sr}_{x} \mathrm{CuO}_{4}$. Finally, a model to study how $A(\mathbf{k}, \omega)$ is affected by thermal fluctuations of the phase, but not the amplitude, of the superconducting order parameter has been treated by Eckl et $a l .{ }^{42}$ for homogeneous systems.

In the present work, we propose a simple model for $A(\mathbf{k}, \omega)$. This model can exhibit a split peak near $\mathbf{k}=(\pi, 0)$, but only for certain parameter choices which are unlikely to be realized experimentally. Our model consists of a BCS superconductor with $d$-wave symmetry, where the pairing field (given by the superconducting order parameter) is inhomogeneous and is also subject to thermal amplitude and phase fluctuations at finite $T$. We assume that those thermal fluctuations are governed by a discretized Ginzburg-Landau (GL) free energy functional. To compute the spectral func- 
tion, we use exact numerical diagonalization of the BCS Hamiltonian on a finite lattice and average over many different configurations of the thermally fluctuating superconducting order parameter as obtained using the Monte Carlo technique. Thermal averages are obtained by averaging over these configurations.

Inhomogeneities are introduced in our model phenomenologically. The atomic lattice is subdivided into cells, which we call $X Y$ cells, of size of $2 \times 2$ atomic sites; within each such cell, we assume $\Delta$ to be constant. Then we choose the coefficients of the GL free energy functional so as to give, at $T=0$, a binary distribution of the superconducting order parameter $|\Delta|$ at each atomic lattice site. $X Y$ cells with small and large $|\Delta|$ values are called $\alpha$ and $\beta$ cells, respectively. We take the distribution of $\alpha$ and $\beta$ cells on the atomic lattice to be random, as suggested by STM experiments. The two parameters which we vary in our calculations are (i) the area fraction $c_{\beta}$ of $\beta$ cells and (ii) the magnitude $\left|\Delta_{\beta}\right|$ of the gap in those cells. The value of $\left|\Delta_{\alpha}\right|$ is kept the same through our calculation and is inferred from the STM experiments.

At $T=0$, we find that the main consequence of this binary, random distribution of $\Delta$ is to broaden the peaks in the spectral function $A(\mathbf{k}, \omega)$ near the points $\mathbf{k}=(\pi, 0)$ [and the symmetry-related points at $\mathbf{k}=(-\pi, 0)$ and $(0, \pm \pi)]$. This broadening is most pronounced at $c_{\beta}=0.5$. For a sufficiently large ratio $\Delta_{\beta} / \Delta_{\alpha}$ of the large to the small gap, we find that $A(\mathbf{k}, \omega)$ near $\mathbf{k}=(\pi, 0)$ shows two peaks rather than one ("split band regime"). Otherwise, we find a single peak which is broadened by disorder. Although the experimental ARPES results of Yoshida et al. ${ }^{43}$ also show a double peak, there are several reasons to believe that this split peak is not caused by the kind of inhomogeneities we consider here. This point is discussed further below.

At finite $T$, we find that, near $\mathbf{k}=(\pi, 0)$, the originally sharp coherence peaks of $A(\mathbf{k}, \omega)$ as a function of $\omega$ broaden and shift to lower energies with increasing $T$. This broadening is similar to that found in the calculations of Eckl et al. ${ }^{42}$ which omits quenched disorder and also includes thermal fluctuations only in the phase but not the amplitude of the gap. These calculations focused on $T$ near that of the phaseordering transition. By contrast, we present calculations showing how $A(\mathbf{k}, \omega)$ evolves near $\mathbf{k}=(\pi, 0)$ as a function of $\omega$ over a broad range of temperature, including both amplitude fluctuations and quenched disorder.

The remainder of this paper is organized as follows. In Sec. II, we briefly describe our model, which is already presented in Ref. 34. In Sec. III, we give our numerical results, followed by a discussion and conclusions in Sec. IV.

\section{MODEL}

\section{A. Microscopic Hamiltonian}

We consider the following Hamiltonian:

$$
H=2 \sum_{\langle i, j\rangle, \sigma} t_{i j} c_{i \sigma}^{\dagger} c_{j \sigma}+2 \sum_{\langle i, j\rangle}\left(\Delta_{i j} c_{i \downarrow} c_{j \uparrow}+\text { c.c. }\right)-\mu \sum_{i, \sigma} c_{i \sigma}^{\dagger} c_{i \sigma} .
$$

Here, $\Sigma_{\langle i, j\rangle}$ denotes a sum over distinct pairs of nearest neighbors on a square lattice with $N$ sites, $c_{j \sigma}^{\dagger}$ creates an electron with spin $\sigma(\uparrow$ or $\downarrow)$ at site $j, \mu$ is the chemical potential, $\Delta_{i j}$ denotes the strength of the pairing interaction between sites $i$ and $j$, and $t_{i j}$ is the hopping energy, which we write as

$$
t_{i j}=-t_{\text {hop }} .
$$

where $t_{\text {hop }}>0$.

Following a similar approach to that of Refs. 34 and 44, we take $\Delta_{i j}$ to be given by

$$
\Delta_{i j}=\frac{1}{4} \frac{\left|\Delta_{i}\right|+\left|\Delta_{j}\right|}{2} e^{i \theta_{i j}}
$$

where

$$
\theta_{i j}= \begin{cases}\left(\theta_{i}+\theta_{j}\right) / 2 & \text { if bond }\langle i, j\rangle \text { is in the } x \text { direction } \\ \left(\theta_{i}+\theta_{j}\right) / 2+\pi & \text { if bond }\langle i, j\rangle \text { is in the } y \text { direction }\end{cases}
$$

and

$$
\Delta_{j}=\left|\Delta_{j}\right| e^{i \theta_{j}}
$$

is the value of the complex superconducting order parameter at site $j$. The sums in Eq. (1) are carried out over a lattice we will refer to as the atomic lattice (as distinguished from the $X Y$ lattice, described below). The first term in Eq. (1) corresponds to the kinetic energy, the second term is a BCS type of pairing interaction with $d$-wave symmetry, and the third term is the energy associated with the chemical potential.

\section{B. Numerical calculation of spectral function}

We wish to compute the spectral function $A(\mathbf{k}, \omega)$ for the system described by the Hamiltonian in Eq. (1). Given the $\Delta_{i}, t_{i j}$, and $\mu, A(\mathbf{k}, \omega)$ is computed through

$$
\mathrm{A}\left(\omega, \mathbf{k},\left\{\Delta_{i}\right\}\right)=\sum_{n, E_{n} \geqslant 0}\left[\left|u_{n}(\mathbf{k})\right|^{2} \delta\left(\omega-E_{n}\right)+\left|v_{n}(\mathbf{k})\right|^{2} \delta\left(\omega+E_{n}\right)\right],
$$

where

$$
\begin{aligned}
& u_{n}(\mathbf{k})=\frac{1}{N^{1 / 2}} \sum_{i=1}^{N} \exp \left(i \mathbf{k} \cdot \mathbf{r}_{i}\right) u_{n}\left(\mathbf{r}_{i}\right), \\
& v_{n}(\mathbf{k})=\frac{1}{N^{1 / 2}} \sum_{i=1}^{N} \exp \left(i \mathbf{k} \cdot \mathbf{r}_{i}\right) v_{n}\left(\mathbf{r}_{i}\right),
\end{aligned}
$$

$E_{n}$ is the $n$th eigenenergy of the Hamiltonian in Eq. (1), and

$$
\psi_{n}\left(\mathbf{r}_{i}\right)=\left[\begin{array}{l}
u_{n}\left(\mathbf{r}_{i}\right) \\
v_{n}\left(\mathbf{r}_{i}\right)
\end{array}\right], \quad i=1, N .
$$

is its $n$th eigenvector, as described in detail in. ${ }^{34}$ Here,

$$
\mathbf{r}_{i}=a_{0}\left(n_{i} \hat{x}+m_{i} \hat{y}\right),
$$

and $n_{i}$ and $m_{i}$ are integers in the ranges $\left[0, N_{x}-1\right]$ and $\left[0, N_{y}-1\right]$. In our numerical calculations, we take the size of the atomic lattice to be $N=N_{x} N_{y}$, where $\hat{x}$ and $\hat{y}$ are unit vectors in the $x$ and $y$ directions, and $a_{0}$ is the lattice con- 
(a)

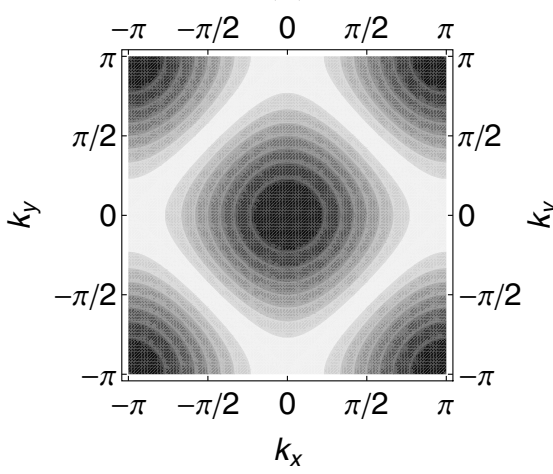

(b)

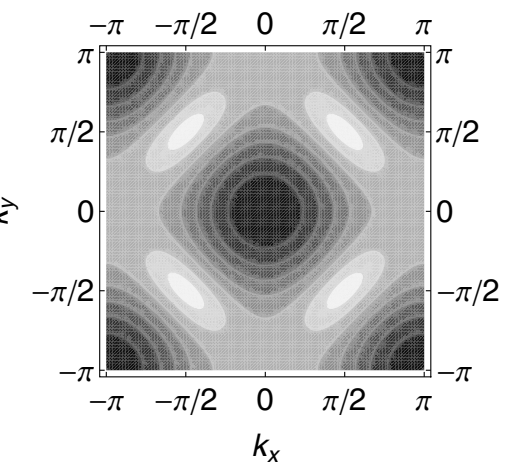

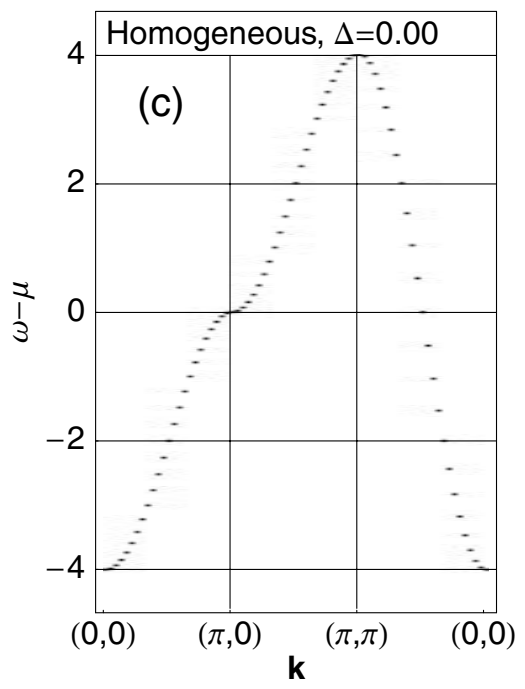

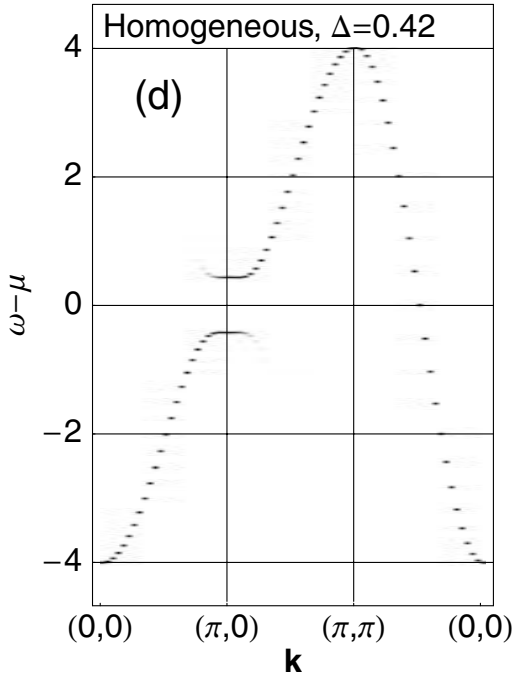

FIG. 1. Contour plots of the energy $E_{\mathbf{k}}$ of single-particle excitations and the corresponding spectral function $A(\mathbf{k}, \omega)$ for two homogeneous systems at zero temperature: one with $\Delta=0$ and the other with $\Delta=0.42$. In parts (a) and (b), the dark (light) regions correspond to large (small) values of the excitation energies, as calculated from Eq. (17). In parts (c) and (d), we plot the positions of the peaks of $A(\mathbf{k}, \omega)$ for these two homogeneous systems.

stant. We use periodic boundary conditions, $\psi_{n}(\mathbf{r})=\psi_{n}(\mathbf{r}$ $\left.+N_{x} a_{0} \hat{x}\right)$ and $\psi_{n}(\mathbf{r})=\psi_{n}\left(\mathbf{r}+N_{y} a_{0} \hat{y}\right)$, which leads to $\mathbf{k}$ vectors of the form

$$
\mathbf{k}=\frac{2 \pi}{a_{0}}\left(\frac{m_{x}}{N_{x}} \hat{x}+\frac{m_{y}}{N_{y}} \hat{y}\right)
$$

The detailed procedure to obtain $\Delta_{i}$ is described in Ref. 34. Basically, we subdivide the atomic lattice into cells, which we call $X Y$ cells, of size $\xi_{0} \times \xi_{0}$. Here, $\xi_{0}$ is the $T=0$ GL coherence length, which we take to be an integer multiple of $a_{0}$. The value of $\Delta_{i}$ is assumed to be the same for each atomic site within a given $X Y$ cell and is governed by the following discretized GL free energy functional:

$$
\begin{aligned}
\frac{F}{K_{1}}= & \sum_{i=1}^{M}\left(\frac{T}{T_{c 0 i}}-1\right) \frac{1}{\lambda_{i}^{2}(0)}\left|\frac{\Delta_{i}}{k_{B} T_{c 0 i}}\right|^{2} \\
& +\sum_{i=1}^{M} \frac{1}{18.76} \frac{1}{\lambda_{i}^{2}(0)}\left|\frac{\Delta_{i}}{k_{B} T_{c 0 i}}\right|^{4} \\
& +\sum_{\langle i j\rangle}\left|\frac{\Delta_{i}}{\lambda_{i}(0) k_{B} T_{c 0 i}}-\frac{\Delta_{j}}{\lambda_{j}(0) k_{B} T_{c 0 j}}\right|^{2} .
\end{aligned}
$$

Here, $K_{1}=\hbar^{4} d /\left[32(9.38) \pi m^{*}, 2 \mu_{B}^{2}\right]$, where $m^{*}=2 m_{e}$ is twice the electron mass, $\mu_{B}$ is the Bohr magneton, and $d$ is the thickness of the superconducting layer. If $d=10 \AA, K_{1}$ $=2866 \mathrm{eV} \AA^{2} . \Delta_{i}$ is the complex gap parameter in the $i$ th $X Y$ cell. In Eq. (12), the sums run over the lattice of $X Y$ cells, each of which contains $\left(\xi / a_{0}\right)^{2}$ atomic sites.

We choose the coefficients of this GL free energy functional $T_{c 0 i}$ and $\lambda_{i}(0)$ to have binary distribution on the $X Y$ lattice, corresponding to either a small or a large value of $\left|\Delta_{i}\right|$. We call an $X Y$ cell with a small (large) value of $\left|\Delta_{i}\right|$ an $\alpha(\beta)$ cell, while the area fraction of $\beta$ cells is called $c_{\beta}$. The corresponding values of $T_{c 0 i}$ and $\lambda_{i}(0)$ are denoted $T_{c 0 \alpha}$ and $T_{c 0 \beta}$. At $T=0$, in a homogeneous system made up entirely of $\alpha(\beta)$ cells, the magnitude of $\left|\Delta_{i}\right|$ will be the same in each $X Y$ cell and given by the minimum of the corresponding free energy functional $F$, i.e., $\left|\Delta_{i}\right|=\sqrt{9.38} k_{B} T_{c 0 \alpha}\left(\sqrt{9.38} k_{B} T_{c 0 \beta}\right)$. In the binary case $\left(0<c_{\beta}<1\right)$, at $T=0$, we will still generally have $\left|\Delta_{i}\right|=\sqrt{9.38} k_{B} T_{c 0 i}$, although this value may be modified slightly by the proximity effect term in $F / K_{1}$ [the last term in Eq. (12)].

We compute $A(\mathbf{k}, \omega)$ at $T=0$ by diagonalizing the model Hamiltonian in Eq. (1) using $\Delta_{i}$ determined by minimizing the Ginzburg-Landau free energy $F$. This minimum value 

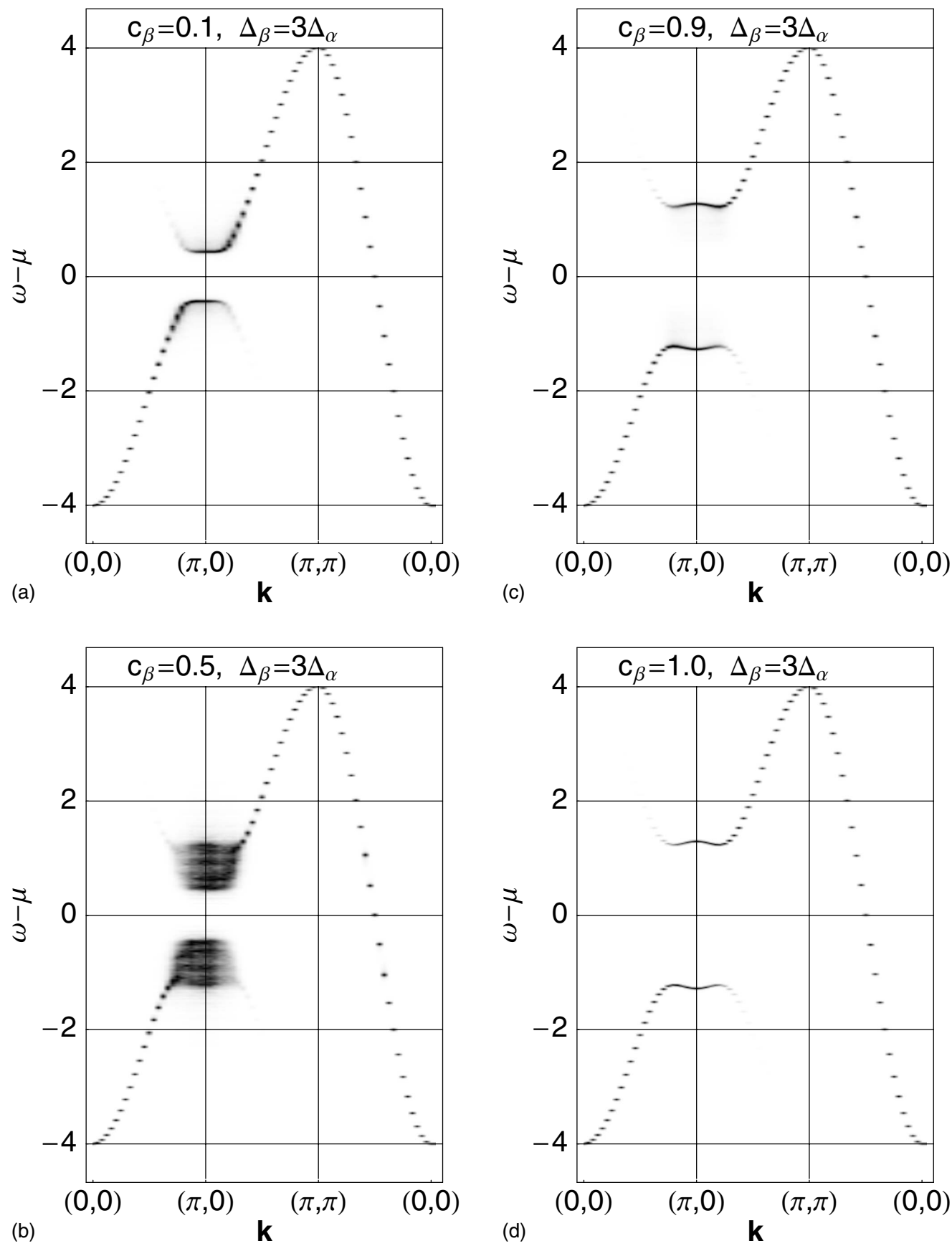

FIG. 2. Same as Figs. 1(c) and 1(d) but for several inhomogeneous systems with different concentrations of $\beta$ (large-gap) cells at $T$ $=0$. In all four plots, atomic sites within the $\alpha$ and $\beta$ cells have $\Delta=0.42$ and $\Delta=1.26$, respectively; the cells are randomly distributed over the atomic lattice, as illustrated in Fig. 4 for the case $c_{\beta}=0.1$. The dark (light) regions correspond to large (small) values of $A(\mathbf{k}, \omega)$. A more quantitative view of $A(\mathbf{k}, \omega)$ is shown in Fig. 3 for $\mathbf{k}=(\pi, 0)$.

will always correspond to gaps $\Delta_{i}=\left|\Delta_{i}\right| e^{i \theta_{i}}$ such that all the phases $\theta_{i}$ are equal. At finite $T$, we compute $A(\mathbf{k}, \omega)$ as an average over different configurations $\left\{\Delta_{i}\right\}$. These are obtained, as in Ref. 34, by assuming that the thermal fluctuations of the $\Delta_{i}$ are governed by the GL free energy functional $F$ described above. Thus, $F$ is treated as an effective classical Hamiltonian fxand thermal averages such as $\langle A(\mathbf{k}, \omega)\rangle$ are computed as

$$
\langle A(\mathbf{k}, \omega)\rangle=\frac{\int \Pi_{i=1}^{N} d^{2} \Delta_{i} e^{-F / k_{B} T} A\left(\mathbf{k}, \omega,\left\{\Delta_{i}\right\}\right)}{\int \Pi_{i=1}^{N} d^{2} \Delta_{i} e^{-F / k_{B} T}} .
$$

We will be using the GL free energy functional at both $T=0$ and finite $T$ in spite of the fact that it was originally 


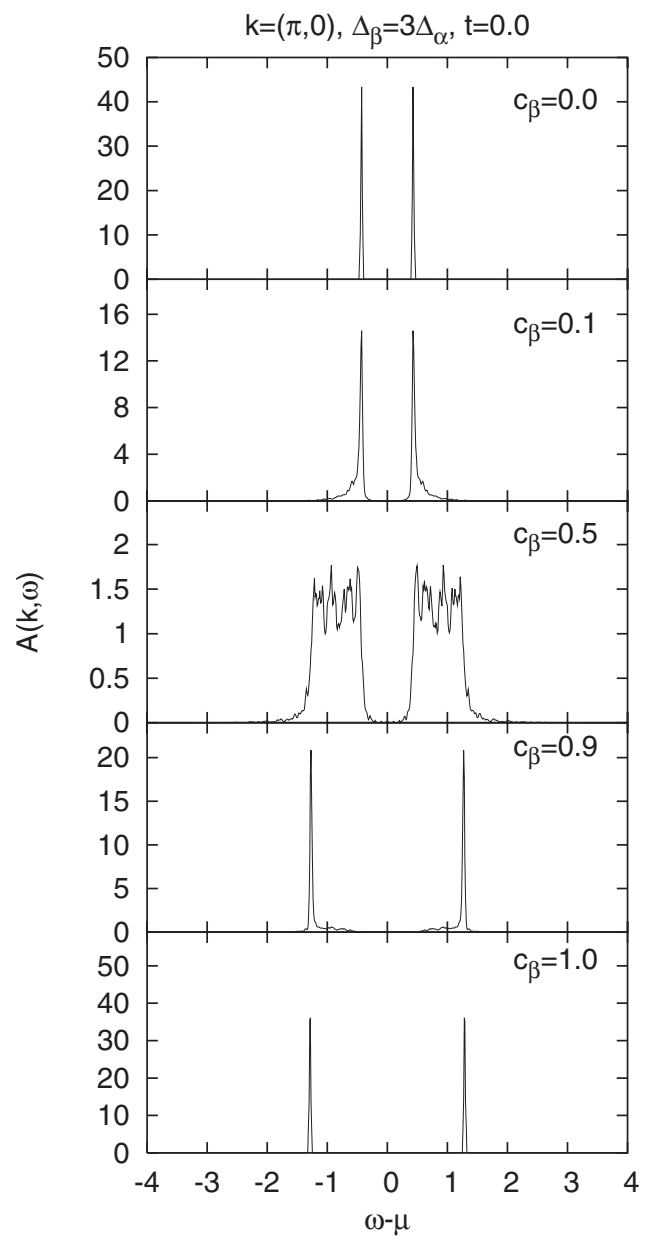

FIG. 3. Spectral function $A[\mathbf{k}=(\pi, 0), \omega]$ as a function of $\omega$ of systems with different concentrations $c_{\beta}$ of $\beta$ cells at zero temperature. We have taken $\Delta_{\alpha}=0.42 t_{\text {hop }}, \Delta_{\beta}=1.26 t_{\text {hop }}$.

intended for $T$ near the mean-field transition temperature. Strictly speaking, the correct free energy functional near $T$ $=0$ should not have the GL form but would be expected to contain additional terms, such as higher powers of $|\psi|^{2}$. We use the GL form for convenience and because we expect that it will exhibit the qualitative behavior that would be seen in a more accurate functional-that is, the effects of inhomogeneities would be qualitatively the same in the GL model as in a more accurate model containing additional powers of $|\psi|^{2}$.

To obtain $A(\mathbf{k}, \omega)$ for a given distribution of the $\Delta_{i}$, we diagonalize the Hamiltonian in Eq. (1) for that configuration then obtain $A(\mathbf{k}, \omega)$ using Eq. (6). The canonical averages are then evaluated using a Metropolis Monte Carlo technique to determine the canonical distribution of the $\Delta_{i}$ at the temperature of interest. The detailed description of this Monte Carlo approach are given in Sec. IV A of Ref. 34. As noted there, we first choose the values of $T_{c 0 i}$ and $\lambda_{i}(0)$ in each $X Y$ cell, taking these to be quenched variables. In contrast to our calculations of Ref. 34, we do not include a smoothing magnetic field to reduce finite-size effects; as a result, our results have more numerical noise than do our earlier results for the density of states.

For the present model calculation, we arbitrarily set the chemical potential $\mu=0$ for simplicity and use $t_{\text {hop }}$ as the unit

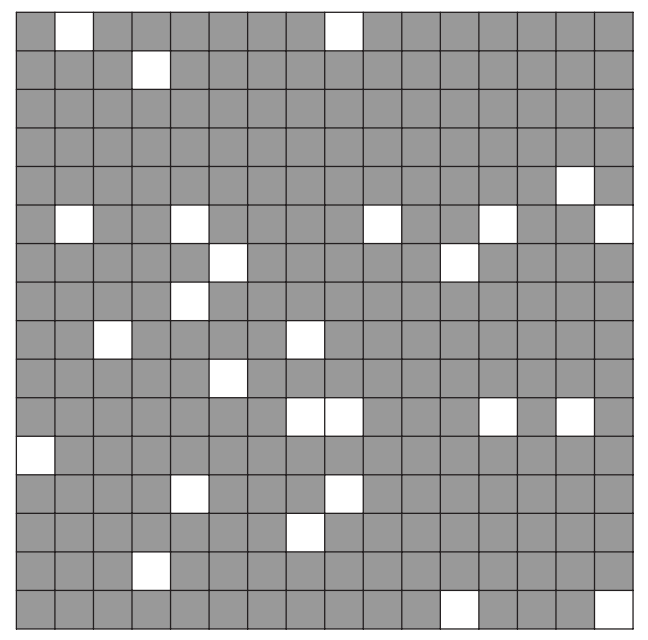

FIG. 4. A typical realization of disorder in a system with a concentration $c_{\beta}=0.1$ of $\beta$ cells (white) immersed in a background of $\alpha$ cells (gray). Each cell contains four atomic sites.

of energy. This corresponds to half-filling in the band model. Exactly half-filling would correspond to $x=0$ in $\mathrm{La}_{x} \mathrm{Sr}_{1-x} \mathrm{CuO}_{4}$ (LSCO), for example. ${ }^{45}$ It should be noted that some of the most interesting experimental results for the spectral function ${ }^{43}$ are carried out in the underdoped superconducting regime of the phase diagram, where $\mu$ is slightly negative. If we set $\mu \neq 0$ in our model, this leads to unequal integrated weights of the spectral function peaks at positive and negative energies, but we have found that, otherwise, our numerical results are not very different from those at $\mu=0$ for our model Hamiltonian. However, the present results and model, for reasons which we discuss below, are probably not directly relevant to those experiments.

In order to show that our results are not strongly affected by setting $\mu=0$, we have also done simulations using $\mu \neq 0$. For example, in Fig. 8 we present results using $\mu=-0.05$. For this value of $\mu$, the average number of electrons per site, defined as

$$
\langle n\rangle=\frac{1}{N} \sum_{i=0}^{N}\left\langle n_{i}\right\rangle,
$$

with

$$
n_{i}=\sum_{\sigma} c_{i, \sigma}^{\dagger} c_{i, \sigma}
$$

is found to be $\langle n\rangle \sim 0.94$. This corresponds to a strongly underdoped cuprate $x \sim 0.06{ }^{46}$

\section{Homogeneous systems}

For a homogeneous system at $T=0, \Delta_{i}=\Delta$ and we can rewrite the Hamiltonian in Eq. (1) as 

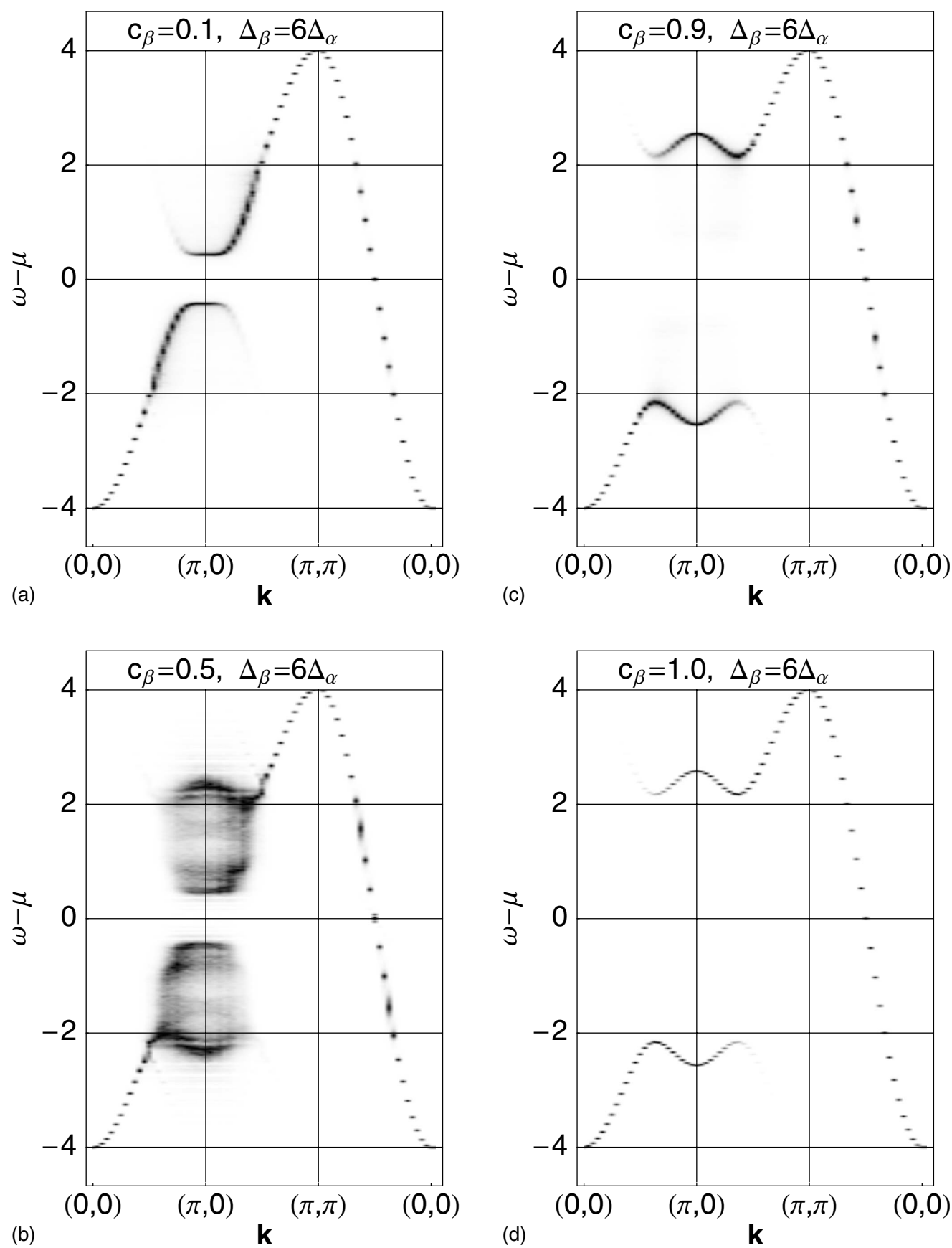

FIG. 5. Same as Fig. 2 except that $\beta$ cells have a $\Delta_{\beta}=2.52$ instead of $\Delta_{\beta}=1.26$.

$$
H=\sum_{\mathbf{k}, \sigma} \epsilon_{\mathbf{k}} c_{\mathbf{k} \sigma}^{\dagger} c_{\mathbf{k} \sigma}+\sum_{\mathbf{k}}\left(\Delta_{\mathbf{k}} c_{\mathbf{k} \downarrow} c_{-\mathbf{k} \uparrow}+\text { c.c. }\right)-\mu \sum_{\mathbf{k}} c_{\mathbf{k} \sigma}^{\dagger} c_{\mathbf{k} \sigma}
$$

where $\epsilon_{\mathbf{k}}=-2 t\left[\cos \left(k_{x} a_{0}\right)+\cos \left(k_{y} a_{0}\right)\right]$ and $\Delta_{\mathbf{k}}=\frac{1}{2} \Delta\left[\cos \left(k_{x} a_{0}\right)\right.$ $\left.-\cos \left(k_{y} a_{0}\right)\right]$. In obtaining Eq. (16), we have used $c_{j}^{\dagger}$ $=1 / N^{1 / 2} \Sigma_{\mathbf{k}} \exp \left(-i \mathbf{k} \cdot \mathbf{r}_{j}\right) c_{\mathbf{k}}^{\dagger}$ and its Hermitian conjugate. In this case, the excitation energies of the system are given by ${ }^{47}$

$$
E_{\mathbf{k}}=\sqrt{\left(\epsilon_{\mathbf{k}}-\mu\right)^{2}+\Delta_{\mathbf{k}}^{2}} \text {. }
$$

The corresponding spectral function will be a sum of two delta functions, as indicated by Eq. (6).

\section{NUMERICAL RESULTS: INHOMOGENEITIES AND THERMAL FLUCTUATIONS}

In this section, we present our numerical results for $A(\mathbf{k}, \omega)$ for inhomogeneous systems both at zero and finite temperatures; for reference, we also show the corresponding results for homogeneous systems in some cases. For $T=0$, we used $48 \times 48$ atomic lattices, while at finite $T$, we used lattices of $32 \times 32$. In all cases, we use $2 \times 2 X Y$ cells. Through the rest of this paper, we show energy measured in units of $t_{\text {hop }}$, distance in units of $a_{0}$, and $\mathbf{k}$ in units of $1 / a_{0}$. 


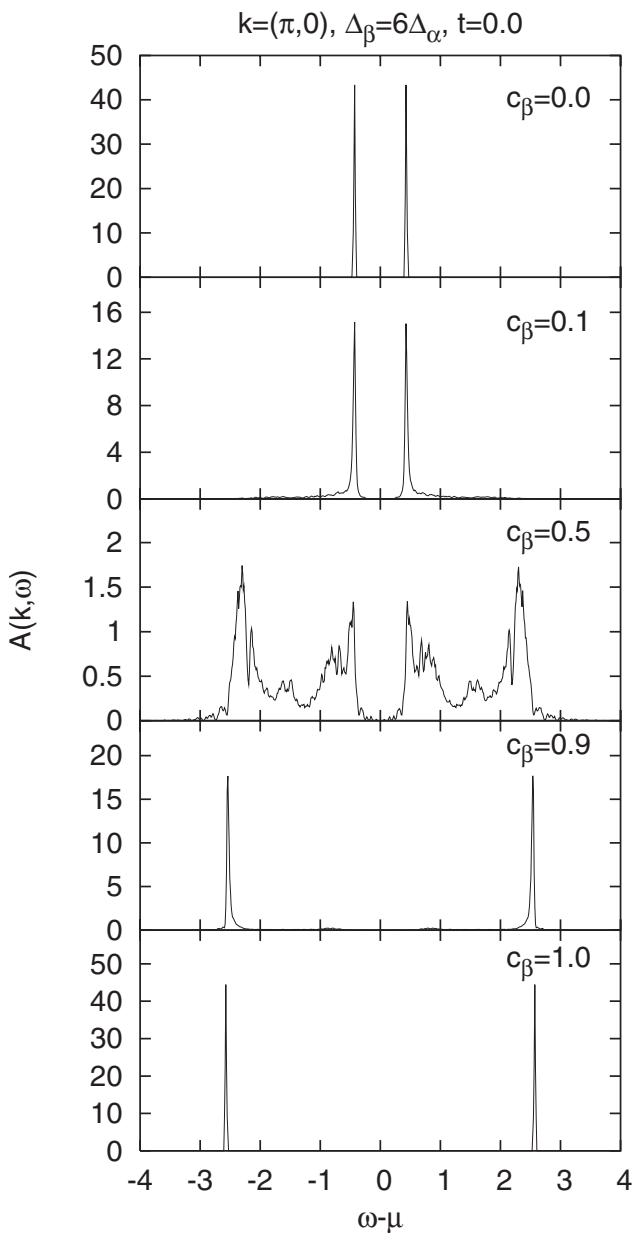

FIG. 6. Same as Fig. 3 but for $\beta$ cells which have $\Delta=2.52$ instead of $\Delta=1.26$.

\section{A. Zero temperature}

Before describing our results at zero temperature, we first comment on our choice of gap parameters used in the calculations. Our primary goal is to ascertain what kinds of qualitative spectral functions could result from the type of inhomogeneity described by our models, not to compare directly to experiment. For this reason, we will examine gaps which are, in general, substantially larger (in units of $t_{h o p}$ ) than those which would describe realistic cuprate superconductors. This point is examined further in Sec IV.

With this preamble, we now present our results at $T=0$. Figure 1 shows the spectral function $A(\mathbf{k}, \omega)$ (represented as a contour plot) as well as plots of the dispersion relation $E_{\mathbf{k}}$ as a function of $\mathbf{k}$ for two homogeneous systems: one with $\Delta=0$ and another with $\Delta=0.42$. For such homogeneous systems, $A(\mathbf{k}, \omega)$ is simply proportional to the sum of two delta functions: $A(\mathbf{k}, \omega) \propto \delta\left(\omega-E_{\mathbf{k}}\right)+\delta\left(\omega+E_{\mathbf{k}}\right)$. In parts (a) and (b), the dark (light) regions correspond to regions where $E_{\mathbf{k}}$, as calculated from Eq. (17), is large (small); these are shown for all $\mathbf{k}$ vectors in the first Brillouin zone (BZ). For a system with $\Delta=0$ [Fig. 1(a)], there are four lines (white) in $\mathbf{k}$ space for which $E_{\mathrm{k}}=0: k_{y}= \pm k_{x} \pm \pi$. When $\Delta>0$ [Fig. 1(b)], the lines are reduced to four points: $\left(k_{x}=\pi / 2, k_{y}= \pm \pi / 2\right)$, and (

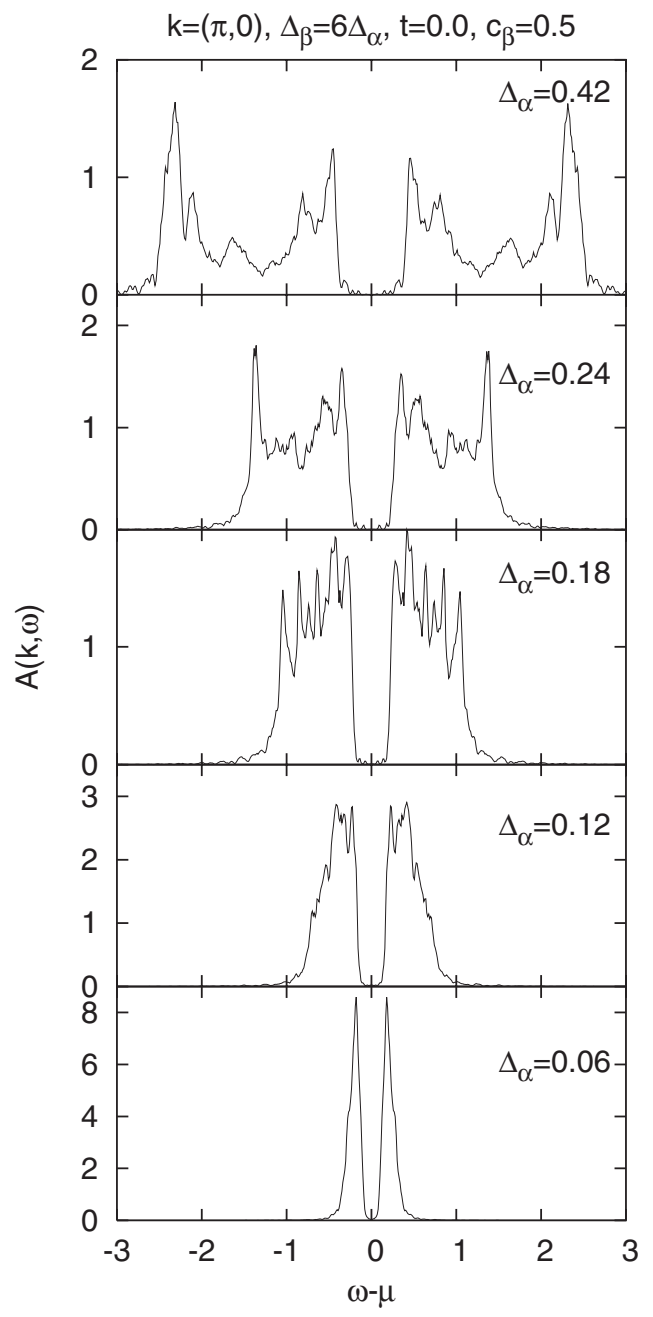

FIG. 7. Spectral function $A[\mathbf{k}=(\pi, 0), \omega]$ as a function of $\omega$ for a system with concentration $c_{\beta}=0.5$ of $\beta$ cells at $T=0$, plotted as a function of the magnitude $\left|\Delta_{\alpha}\right|$ of the component with the smaller gap. In all cases, $\left|\Delta_{\beta} / \Delta_{\alpha}\right|=6$.

$k_{x}=-\pi / 2, k_{y}= \pm \pi / 2$ ), located at the center of the white blobs in Fig. 1(b). In Figs. 1(c) and 1(d), density plots of $A(\mathbf{k}, \omega)$ as a function of $\omega$ are presented for those homogeneous systems at selected $\mathbf{k}$ values. These values lie along three standard lines in the first BZ: from $\mathbf{k}=(0,0)$ to $\mathbf{k}$ $=(\pi, 0)$, from $\mathbf{k}=(\pi, 0)$ to $\mathbf{k}=(\pi, \pi)$, and from $\mathbf{k}=(\pi, \pi)$ to $\mathbf{k}=(0,0)$. Dark (light) regions correspond to large (small) values of the spectral function. For each $\mathbf{k}$ in these homogeneous systems, there is a sharp peak in $A(\mathbf{k}, \omega)$, whose energy and width are indicated as the very short dashed lines in the plot. Also, the spectral function is clearly most strongly affected by a finite value of $\Delta$ near $\mathbf{k}=(\pi, 0)$, where an energy gap of magnitude $\Delta$ opens around $\omega-\mu=0$.

Figures 2 and 3 show the spectral function of several inhomogeneous systems with different concentrations $c_{\beta}$ of $\beta$ cells at $T=0$. In these systems, the atomic cells within the $\beta$ cells have $\Delta=1.26$ and are randomly distributed in the atomic lattice, while $\alpha$ cells, which occupy the rest of the lattice, have $\Delta=0.42$. Figure 4 shows representative arrangements of $\alpha$ and $\beta$ cells for a $16 \times 16 X Y$ lattice with $c_{\beta}$ 
$=0.1$. We can observe in Fig. 2 that the disorder introduced by this binary distribution of the superconducting order parameter affects mostly the region $\mathbf{k}=(\pi, 0)$. This disorder effect is almost unobservable for $c_{\beta}=0.9$; the results are almost the same as those for $c_{\beta}=1.0$, a homogeneous system with only $\beta$ cells. On the other hand, a small but noticeable disorder effect is observed for $c_{\beta}=0.1$, in the form of a slight broadening of the spectral function at $\mathbf{k} \simeq(0.8 \pi, 0)$. However, it is the system with $c_{\beta}=0.5$ that shows a most dramatic blurring of the energy in the region of $\mathbf{k}=(\pi, 0)$, as we will now discuss.

Since the effects of the binary distribution of $\Delta$ are more pronounced near $\mathbf{k}=(\pi, 0)$, we have also plotted $A(\mathbf{k}, \omega)$ versus $\omega$ for fixed $\mathbf{k}=(\pi, 0)$ at different values of $c_{\beta}$ in Fig. 3. For the pure $\alpha$ system, $c_{\beta}=0.0$, two sharp peaks appear at $|\omega|=\Delta_{\alpha}=0.42$. When a fraction 0.1 of the $\alpha X Y$ cells are replaced by $\beta$ cells, $c_{\beta}=0.1$, the height of the peaks decreases from about 45 (arbitrary units) to about 15 , with a corresponding broadening of the peak and a shifting of the weight toward a higher energy. At $c_{\beta}=0.5$, the peak height is only about 1.5 , and the width is very large; the peak fills the entire frequency range from $\omega=\Delta_{\alpha}=0.42$ to $\Delta_{\beta}=1.26$. At $c_{\beta}=0.9$, most of the weight of $A(\mathbf{k}, \omega)$ shifts to $\omega=\Delta_{\beta}$ $=1.26$, with a slight broadening near the bottom of the peaks, which is, however, less pronounced than the corresponding broadening of the $c_{\beta}=0.1$ peaks. At $c_{\beta}=1.0$, the peaks become sharp at $\omega= \pm \Delta_{\beta}= \pm 1.26$.

We now discuss the calculated effects of a binary gap distribution on systems similar to those of Fig. 2 but with $\Delta_{\beta}=2.52$ instead of $\Delta_{\beta}=1.26$ (see Figs. 5 and 6). These systems, like the one previously discussed, have $\Delta_{\alpha}=0.42$. For concentrations $c_{\beta}=0.1$ and $c_{\beta}=0.9$, the effect of inhomogeneities qualitatively resembles that seen for $\Delta_{\beta}=1.26$; they produce broadening of the spectral function near $\mathbf{k}=(\pi, 0)$. The main difference is that the broadening is slightly greater for $\Delta_{\beta}=2.52$. However, the case $c_{\beta}=0.5$ shows a real qualitative change; the spectral function now splits into two welldefined peaks for $\mathbf{k}$ vectors near $(\pi, 0)$. We can better visualize this effect by looking at Fig. 6, where we plot $A(\mathbf{k}, \omega)$ versus $\omega$ for fixed $\mathbf{k}=(\pi, 0)$ and several values of $c_{\beta}$. Clearly, $A(\mathbf{k}, \omega)$ for $c_{\beta}=0.1$ and $c_{\beta}=0.9$ behaves similarly to the case $\Delta_{\beta}=1.26$ : slightly broadened peaks at an energy near the $\Delta$ of the majority of the $X Y$ cells, i.e., at $\omega=0.42$ for $c_{\beta}=0.1$ and at $\omega=2.52$ for $c_{\beta}=0.9$. However, for $c_{\beta}=0.5, A(\mathbf{k}, \omega)$ shows several peaks, two of which are particularly clear: one at $\omega \simeq 0.42$ and the other at $\omega \simeq 2.52$. This is the split band regime one expects for large contrast between $\Delta_{\alpha}$ and $\Delta_{\beta}$.

In order to better visualize how the spectral function depends on disorder, we have calculated $A(\mathbf{k}, \omega)$ as a function of $\left|\Delta_{\alpha}\right|$ for a fixed ratio $\left|\Delta_{\beta} / \Delta_{\alpha}\right|=6$ at $c_{\beta}=0.5$. The results are shown in Figs. 7 and 8. This series of plots clearly shows the evolution of $A(\mathbf{k}, \omega)$ from a split band regime at $\left|\Delta_{\beta}\right|=2.52$ (in units of $t_{\text {hop }}$ ) to a broadened single band for $\left|\Delta_{\beta}\right|$ $\left.=0.22 \mid t_{h o p}\right)$ or smaller. In general, we find that the split band regime occurs only if the difference $\left|\Delta_{\beta}\right|-\left|\Delta_{\alpha}\right|$ is of order $t_{\text {hop }}$ or larger; otherwise, $A(\mathbf{k}, \omega)$ at $\mathbf{k}=(\pi, 0)$ is simply the sum of two broadened peaks at positive and negative energies.

\section{B. Finite temperatures}

Figure 9 shows the $T$ dependence of $A(\mathbf{k}, \omega)$ for a system with $c_{\beta}=0.1$. The $\alpha$ and $\beta$ cells are now characterized by

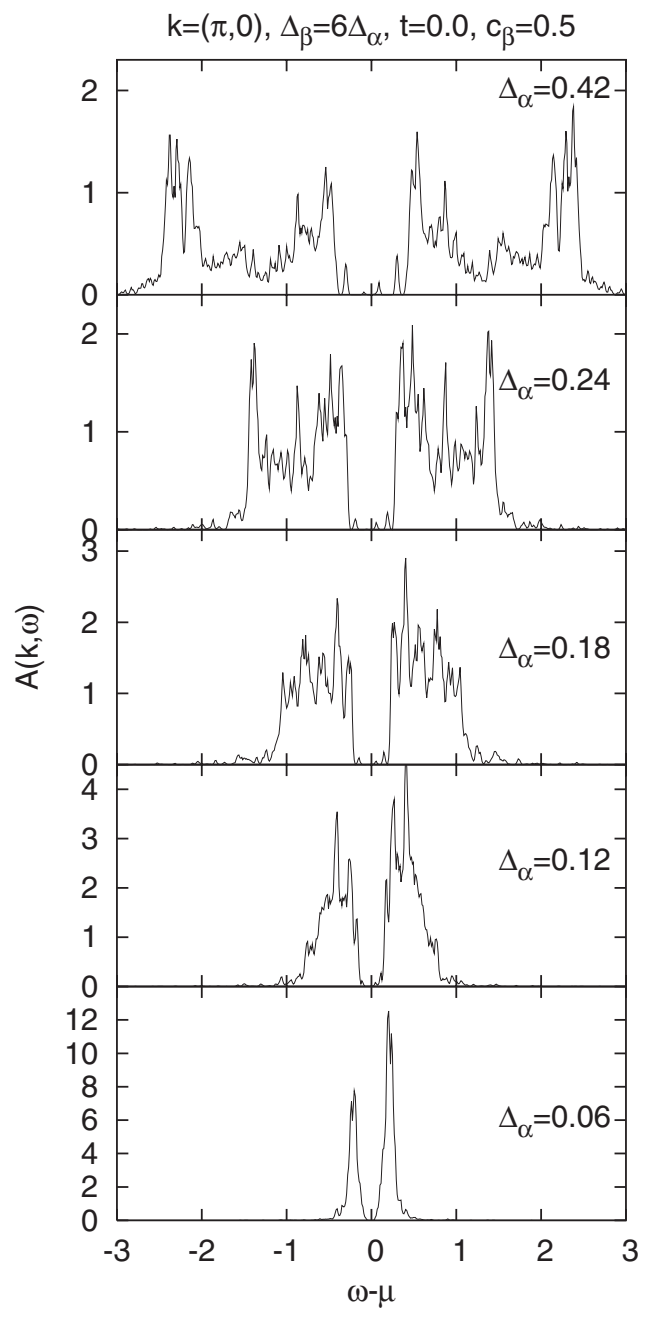

FIG. 8. Same as Fig. 7 but with chemical potential $\mu=-0.05$ instead of $\mu=0$. With our model, setting $\mu=-0.05$ results in having an average occupation number $\langle n\rangle \sim 0.94$, which corresponds to a strongly underdoped cuprate $x \sim 0.06$.

values of $t_{c 0}$ such that at low $T, \Delta=0.42$ in the $\alpha$ cells and 1.26 in the $\beta$ cells. The value of $\Delta$ itself at finite $T$ will, of course, thermally fluctuate, as governed by the GL free energy functional discussed at the end of Sec. II. The spectral function presented here is therefore an average of $A\left(\omega, \mathbf{k},\left\{\Delta_{i}\right\}\right)$ over different configurations $\left\{\Delta_{i}\right\}$ obtained by a Monte Carlo sampling procedure, as described above and in Ref. 34. Hereafter, we denote this ensemble average simply as $A(\mathbf{k}, \omega)$.

Figure 9 shows that, as in the case of quenched disorder, $A(\mathbf{k}, \omega)$ is most strongly affected by thermal fluctuations near $\mathbf{k}=(\pi, 0)$, where it broadens more and more with increasing $T$. In addition to this broadening, the peaks can be seen to shift toward smaller energies. This behavior can be seen more clearly in Fig. 10, which shows $A(\mathbf{k}, \omega)$ at $\mathbf{k}=(\pi, 0)$ as a function of $\omega-\mu$. We observe that at $T=0, A(\mathbf{k}, \omega)$ shows relatively sharp peaks at $\omega-\mu= \pm 0.42$, with some disorderinduced broadening only in the wings of the peak. At $t$ $=0.01$, the peak height of $A(\mathbf{k}, \omega)$ decreases from $\sim 17$ to about $\sim 7$, with a correspondingly increased width. 

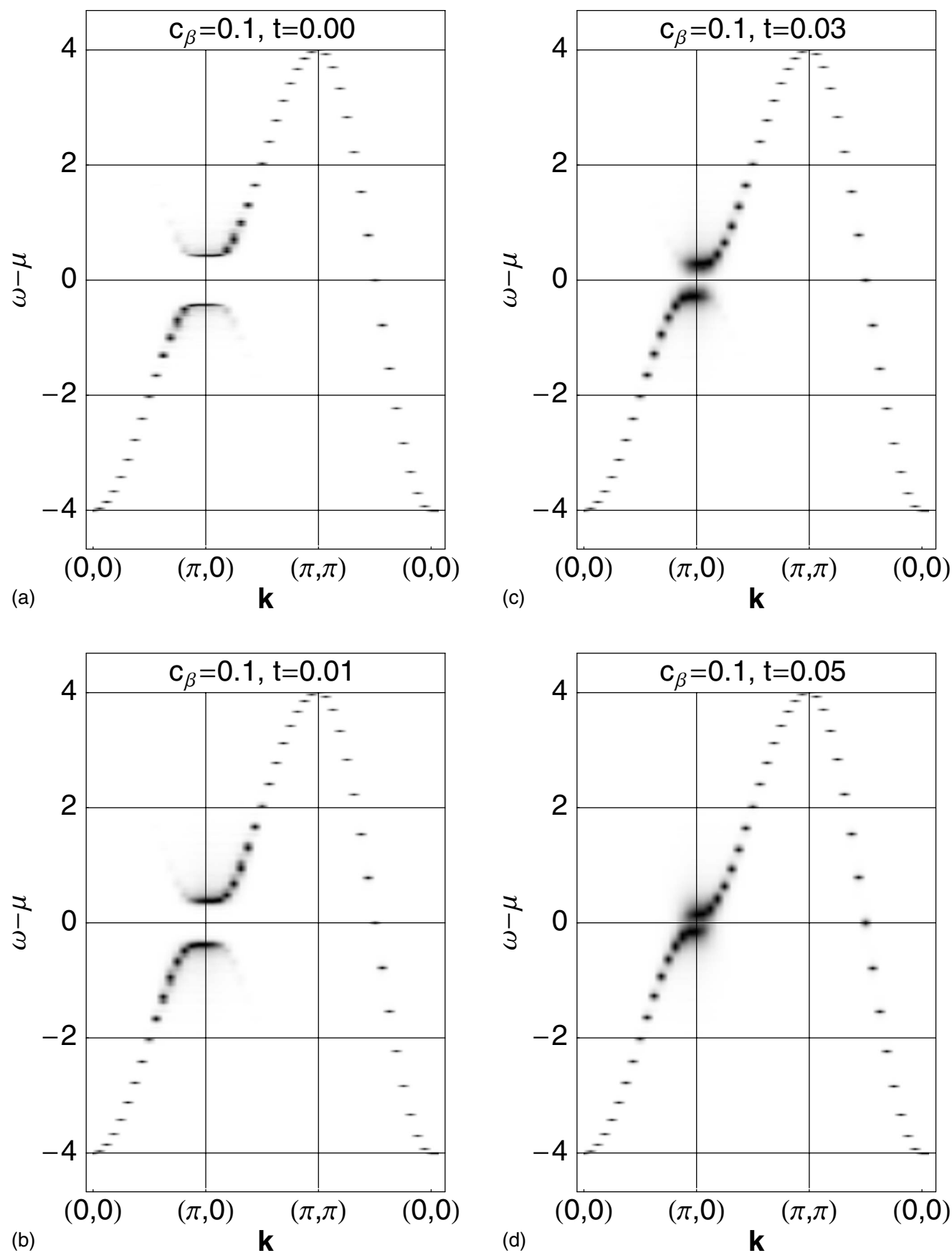

FIG. 9. Plots of the spectral function $A(\mathbf{k}, \omega)$ for a system at zero and finite temperatures. The system has a concentration $c_{\beta}=0.1$ of $\beta$ cells with $\Delta_{\beta}=1.26$ randomly distributed in a background of $\alpha$ cells having $\Delta_{\alpha}=0.42$. The phase-ordering temperature is $t_{c} \approx 0.035$. The detailed evolution of the curve $A[\mathbf{k}=(\pi, 0), \omega]$ versus $\omega$ can be seen in Fig. 10.

As the temperature is increased, the system eventually undergoes a phase-disordering transition, above which the superconductor loses phase coherence. For the parameters used in Fig. 10, this transition occurs at $t_{c} \simeq 0.035$. $t_{c}$ is the phase-ordering transition temperature in units of $t_{h o p} / k_{B}$. We use a dimensionless temperature $t=k_{B} T / t_{\text {hop }}$ in these plots. At $t=0.03$, near but slightly below the phase-ordering temperature $t_{c} \simeq 0.035$, the height of the peak is further decreased, its width further increased, and its energy shifted to a still lower energy. At $t=0.05>t_{c}$, the peak shifts still further toward lower energy, but the maximum remains at finite energy.

\section{DISCUSSION}

We have presented a simple model to study how the spectral function of a model $d$-wave superconductor is affected by quenched inhomogeneities and by thermal fluctuations of the superconducting order parameter. The model consists of a BCS Hamiltonian for an order parameter with $d_{x^{2}-y^{2}}$-wave symmetry, which has a position-dependent pairing field and which also undergoes finite-temperature thermal fluctuations. The spatial dependence we assume for the pairing field is motivated by recent STM experiments on Bi2212; we as- 


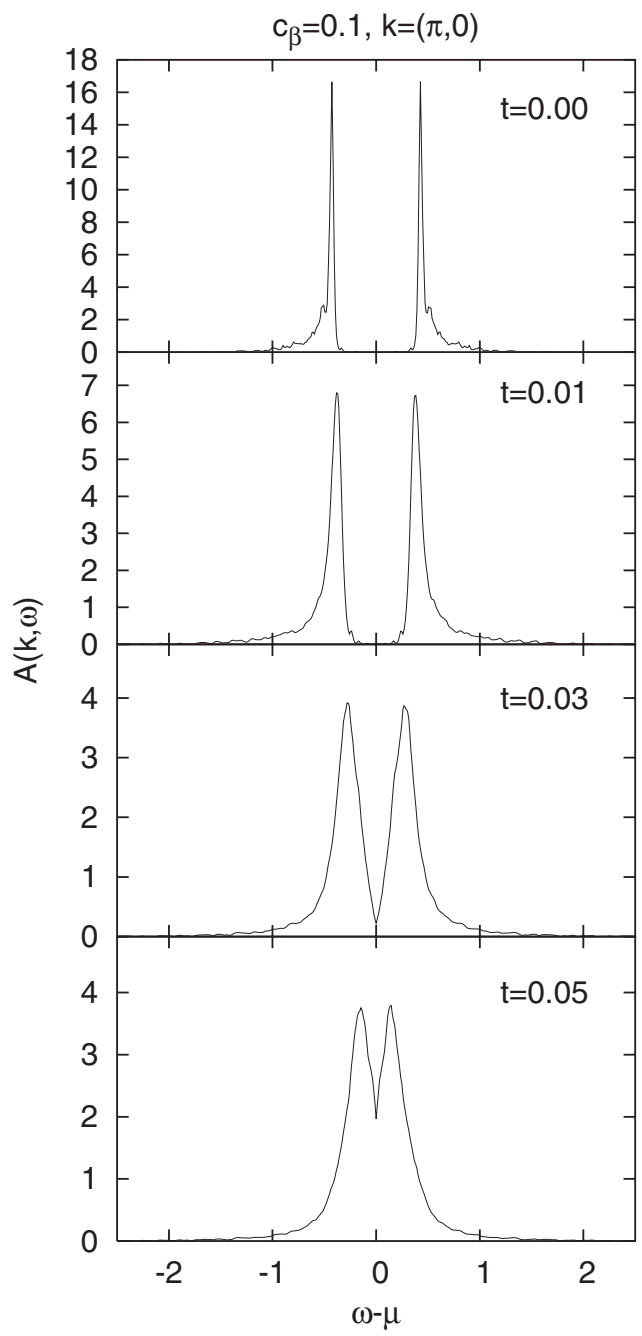

FIG. 10. Spectral function $A[\mathbf{k}=(\pi, 0), \omega]$ as a function of $\omega$ for a system with $c_{\beta}=0.1$ at different temperatures. The phase-ordering temperature of the system is $t_{c} \simeq 0.035$.

sume two types of regions: an $\alpha$ region with a small gap and a $\beta$ region with a large gap. To treat thermal fluctuations (of both amplitude and phase of the superconducting order parameter), we assume that they are governed by a suitable Ginzburg-Landau free energy function, which we treat by classical Monte Carlo simulations.

At $T=0$, we find that $A(\mathbf{k}, \omega)$ is most strongly affected by disorder near $\mathbf{k}=(\pi, 0)$. In general, this effect consists of a broadening of the peaks of $A(\mathbf{k}, \omega)$ (plotted as a function of $\omega$ for fixed k). However, at area fraction $c_{\beta}=0.5$, we find that quenched disorder can have two qualitatively different effects, depending on the relative magnitudes of $\Delta_{\alpha}$ and $\Delta_{\beta}$. If the difference between $\Delta_{\alpha}$ and $\Delta_{\beta}$ is small, $A(\mathbf{k}, \omega)$ has a single, broad peak for $\mathbf{k}$ near $(\pi, 0)$, extending from $\sim \Delta_{\alpha}$ to $\sim \Delta_{\beta}$. However, for a large enough difference between $\Delta_{\alpha}$ and $\Delta_{\beta}$, the $A(\mathbf{k}, \omega)$ show a characteristic split band behavior; instead of a wide, single peak, there are two prominent peaks, at $\omega=\Delta_{\alpha}$ and $\omega=\Delta_{\beta}$.

Thermal fluctuations of the pairing field also have their strongest effect on $A(\mathbf{k}, \omega)$ near $\mathbf{k}=(\pi, 0)$. The effect consists of a gradual broadening of the $T=0$ peaks with increasing temperature and also a shifting of those peaks toward lower energies. However, no dramatic change is noticeable near the phase-ordering transition.

Finally, we comment on the possible connection, if any, between our results and experiment. In recent angle-resolved photoemission studies by Yoshida et al. ${ }^{43}$ for LSCO, it was observed that for doping level $x=0.03$, a second branch developed in the dispersion relation near $\mathbf{k}=(\pi, 0)$. An explanation for the presence of these two branches has recently been suggested by Mayr et al. ${ }^{41}$ These authors showed that the extra branch could be explained by a model with quenched disorder, in which the material breaks up into spatially separated superconducting and antiferromagnetic patches.

In the present work, we find that a similar effect, with two spectral peaks, can be produced if there are spatially distinct superconducting regions with sufficiently different superconducting gaps. However, we also find that a split spectral peak can be produced only if the magnitudes of the gaps $\left|\Delta_{\alpha}\right|$ and $\left|\Delta_{\beta}\right|$ and of their difference are much larger than seems physically reasonable. Specifically, unless one of the gaps in the bimodal distribution is around $2.5 t_{\text {hop }}$, we do not obtain a split peak in $A(\mathbf{k}, \omega)$ at the point $\mathbf{k}=(\pi, 0)$. For typical values $\left(t_{\text {hop }} \sim 200 \mathrm{meV}\right)$, this would represent a $|\Delta|$ of around $0.5 \mathrm{eV}$. Since the average value of $|\Delta|$ in most of the cuprate superconductors is $\sim 0.05 \mathrm{eV}$, it seems most unlikely that random spatial fluctuations in $|\Delta|$, due to quenched disorder, could produce such a large gap locally. Furthermore, even with such large quenched fluctuations in the gap, we need a bimodal gap distribution to obtain a split spectral functionequally large quenched fluctuations but with a continuous distribution due to quenched disorder would probably not give rise to a split spectral function. Therefore, it seems very improbable that our model could account for the second branch in the dispersion relation reported in Ref. 43. However, our results should give a reasonable picture of how quenched gap inhomogeneities affect $A(\mathbf{k}, \omega)$ in a $d$-wave superconductor over a range of parameters.

\section{ACKNOWLEDGMENTS}

We are grateful for support through National Science Foundation Grant No. DMR04-13395. We also thank Rajdeep Sensarma for useful conversations. The computations described here were carried out using the facilities of the Ohio Supercomputing Center, with the help of a grant of time. 
*Present address: Department of Physics and Astronomy, University of Rochester, Rochester, NY 14627; valdez@pas.rochester.edu

†stroud@mps.ohio-state.edu

${ }^{1}$ T. Cren, D. Roditchev, W. Sacks, J. Klein, J.-B. Moussy, C. Deville-Cavellin, and M. Lagues, Phys. Rev. Lett. 84, 147 (2000).

${ }^{2}$ C. Howald, P. Fournier, and A. Kapitulnik, Phys. Rev. B 64, 100504(R) (2001).

${ }^{3}$ S. H. Pan, J. P. O'Neal, R. L. Badzey, C. Chamon, H. Ding, J. R. Engelbrecht, Z. Wang, H. Eisaki, S. Uchida, A. K. Gupta, K.-W. Ng, E. W. Hudson, K. M. Lang, and J. C. Davis, Nature (London) 413, 282 (2001)

${ }^{4}$ K. M. Lang, V. Madhavan, J. E. Hoffman, E. W. Hudson, H. Eisaki, S. Uchida, and J. C. Davis, Nature (London) 415, 412 (2002).

${ }^{5}$ C. Howald, H. Eisaki, N. Kaneko, M. Greven, and A. Kapitulnik, Phys. Rev. B 67, 014533 (2003).

${ }^{6}$ T. Kato, S. Okitsu, and H. Sakata, Phys. Rev. B 72, 144518 (2005).

${ }^{7}$ A. C. Fang, L. Capriotti, D. J. Scalapino, S. A. Kivelson, N. Kaneko, M. Greven, and A. Kapitulnik, Phys. Rev. Lett. 96, 017007 (2006).

${ }^{8}$ H. Mashima, N. Fukuo, Y. Matsumoto, G. Kinoda, T. Kondo, H. Ikuta, T. Hitosugi, and T. Hasegawa, Phys. Rev. B 73, 060502(R) (2006)

${ }^{9}$ S.-W. Cheong, G. Aeppli, T. E. Mason, H. Mook, S. M. Hayden, P. C. Canfield, Z. Fisk, K. N. Clausen, and J. L. Martinez, Phys. Rev. Lett. 67, 1791 (1991).

${ }^{10}$ K. Yamada, C. H. Lee, K. Kurahashi, J. Wada, S. Wakimoto, S. Ueki, H. Kimura, Y. Endoh, S. Hosoya, G. Shirane, R. J. Birgeneau, M. Greven, M. A. Kastner, and Y. J. Kim, Phys. Rev. B 57, 6165 (1998).

${ }^{11}$ J. M. Tranquada, B. J. Sternlieb, J. D. Axe, Y. Nakamura, and S. Uchida, Nature (London) 375, 561 (1995).

${ }^{12}$ T. Niemöller, N. Ichikawa, T. Frello, H. Hünnefeld, N. H. Andersen, S. Uchida, J. R. Schneider, and J. M. Tranquada, Eur. Phys. J. B 12, 509 (1999).

${ }^{13}$ H. A. Mook, Pengcheng Dai, S. M. Hayden, G. Aeppli, T. G. Perring, and F. Dogan, Nature (London) 395, 580 (1998).

${ }^{14}$ M. Arai, T. Nishijima, Y. Endoh, T. Egami, S. Tajima, K. Tomimoto, Y. Shiohara, M. Takahashi, A. Garrett, and S. M. Bennington, Phys. Rev. Lett. 83, 608 (1999).

${ }^{15}$ Pengcheng Dai, H. A. Mook, R. D. Hunt, and F. Dogan, Phys. Rev. B 63, 054525 (2001).

${ }^{16}$ S. M. Hayden, H. A. Mook, Pengcheng Dai, T. G. Perring, and F. Dogan, Nature (London) 429, 531 (2004).

${ }^{17}$ X. F. Sun, Y. Kurita, T. Suzuki, Seiki Komiya, and Yoichi Ando, Phys. Rev. Lett. 92, 047001 (2004).

${ }^{18}$ T. Hanaguri, C. Lupien, Y. Kohsaka, D.-H. Lee, M. Azuma, M. Takano, H. Takagi, and J. C. Davis, Nature (London) 430, 1001 (2004).

${ }^{19}$ Jan Zaanen and Olle Gunnarsson, Phys. Rev. B 40, 7391 (1989); Kazushige Machida, Physica C 158, 192 (1989); M. Kato, K. Machida, H. Nakanishi, and M. Fujita, J. Phys. Soc. Jpn. 59, 1047 (1990);

${ }^{20}$ V. J. Emery, S. A. Kivelson, and H. Q. Lin, Phys. Rev. Lett. 64,
475 (1990)

${ }^{21}$ V. J. Emery and S. A. Kivelson, Physica C 209, 597 (1993).

${ }^{22}$ U. Löw, V. J. Emery, K. Fabricius, and S. A. Kivelson, Phys. Rev. Lett. 72, 1918 (1994).

${ }^{23}$ See V. J. Emery, S. A. Kivelson, and J. M. Tranquada, Proc. Natl. Acad. Sci. U.S.A. 96, 8814 (1999).

${ }^{24}$ Reza Jamei, Steven Kivelson, and Boris Spivak, Phys. Rev. Lett. 94, 056805 (2005).

${ }^{25}$ Daniel Valdez-Balderas and David Stroud, Phys. Rev. B 72, 214501 (2005).

${ }^{26}$ Ivar Martin and Alexander V. Balatsky, Physica C 357-360, 46 (2001).

${ }^{27}$ Ziqiang Wang, Jan R. Engelbrecht, Shancai Wang, Hong Ding, and Shuheng H. Pan, Phys. Rev. B 65, 064509 (2002).

${ }^{28}$ W. A. Atkinson, Phys. Rev. B 71, 024516 (2005).

${ }^{29}$ Tamara S. Nunner, Brian M. Andersen, Ashot Melikyan, and P. J. Hirschfeld, Phys. Rev. Lett. 95, 177003 (2005).

${ }^{30}$ A. Ghosal, M. Randeria, and N. Trivedi, Phys. Rev. Lett. 81, 3940 (1998); Phys. Rev. B 65, 014501 (2001).

${ }^{31}$ Ming Cheng and W. P. Su, Phys. Rev. B 72, 094512 (2005).

${ }^{32}$ Matthias Mayr, Gonzalo Alvarez, Adriana Moreo, and Elbio Dagotto, Phys. Rev. B 73, 014509 (2006).

${ }^{33}$ R. Jamei, J. Robertson, E.-A. Kim, A. Fang, A. Kapitulnik, and S. A. Kivelson, Phys. Rev. B 74, 174521 (2006).

${ }^{34}$ Daniel Valdez-Balderas and David Stroud, Phys. Rev. B 74, 174506 (2006).

${ }^{35}$ Katsunori Wakabayashi, T. M. Rice, and Manfred Sigrist, Phys. Rev. B 72, 214517 (2005).

${ }^{36}$ P. Pieri, L. Pisani, and G. C. Strinati, Phys. Rev. Lett. 92, 110401 (2004).

${ }^{37}$ Marc G. Zacher, Robert Eder, Enrico Arrigoni, and Werner Hanke, Phys. Rev. B 65, 045109 (2002).

${ }^{38}$ Arun Paramekanti, Mohit Randeria, and Nandini Trivedi, Phys. Rev. Lett. 87, 217002 (2001).

${ }^{39}$ Qijin Chen, K. Levin, and Ioan Kosztin, Phys. Rev. B 63, 184519 (2001).

${ }^{40}$ Takashi Hotta, Matthias Mayr, and Elbio Dagotto, Phys. Rev. B 60, 13085 (1999).

${ }^{41}$ Matthias Mayr, Gonzalo Alvarez, Adriana Moreo, and Elbio Dagotto, Phys. Rev. B 73, 014509 (2006).

${ }^{42}$ T. Eckl, W. Hanke, S. V. Borisenko, A. A. Kordyuk, T. Kim, A. Koitzsch, M. Knupfer, and J. Fink, Phys. Rev. B 70, 094522 (2004).

${ }^{43}$ T. Yoshida, X. J. Zhou, T. Sasagawa, W. L. Yang, P. V. Bogdanov, A. Lanzara, Z. Hussain, T. Mizokawa, A. Fujimori, H. Eisaki, Z.-X. Shen, T. Kakeshita, and S. Uchida, Phys. Rev. Lett. 91, 027001 (2003).

${ }^{44}$ T. Eckl, D. J. Scalapino, E. Arrigoni, and W. Hanke, Phys. Rev. B 66, 140510(R) (2002).

${ }^{45} x=0$ is actually in the antiferromagnetic regime of LSCO. However, our model does not contain any antiferromagnetic interactions, and thus should behave similarly to $\mu$ slightly negative, which corresponds to strongly underdoped LSCO.

${ }^{46}$ Andrea Damascelli, Zahid Hussain, and Zhi-Xun Shen, Rev. Mod. Phys. 75, 473 (2003).

${ }^{47}$ See, e.g., Michael Tinkham, Introduction to Superconductivity, 2nd ed. (Dover, Mineola, 1996). 